\title{
ERRATA
}

\section{Purification and Properties of a New $\beta$-Lactamase from Pseudomonas cepacia}

K. HIRAI, S. IYOBE, M. INOUE, AND S. MITSUHASHI

Department of Microbiology, School of Medicine, Gunma University, Maebashi, Japan

Vol. 17, no. 3, p. 357: Table 2 was printed incorrectly. The following is the corrected version of the table:

TABLE 2. Kinetics of hydrolysis of various cephalosporins and penicillins by $\beta$-lactamase from

\begin{tabular}{lcrr}
\multicolumn{3}{c}{$P$ cepacia $G N 11164$} \\
\hline \multicolumn{1}{c}{ Substrate } & $K_{m}(\mu \mathrm{M})$ & $K_{i}(\mu \mathrm{M})^{a}$ & $V_{\max }{ }^{b}$ \\
\hline Cephaloridine & 70.4 & & 62 \\
Cefazolin & 40.0 & & 97 \\
Cephalothin & 71.4 & & 200 \\
Cephalexin & 30.8 & & 38 \\
Cefsulodin & 500 & & 100 \\
Cefotiam & 133.3 & & 100 \\
Cefamandole & 181.8 & & 280 \\
Cefuroxime & 105.2 & & 148 \\
Cefotaxime & 250 & & 108 \\
FK 749 & 100 & & 7 \\
Cefoperazone & & 5.36 & 6 \\
Cefoxitin & & & $<1$ \\
Cefmetazole & & & $<1$ \\
Benzylpenicillin & 46.5 & & 100 \\
Ampicillin & 133.3 & & 200 \\
Carbenicillin & 17.0 & & 22 \\
Cloxacillin & & 3.36 & \\
Methicillin & & 2.27 & \\
Clavulanic acid & & 1.72 & \\
CP 45899 & & 1.82 & \\
\hline
\end{tabular}

${ }^{a}$ Values of $K_{i}$ were determined with CET as a substrate.

${ }^{b}$ Rates of hydrolysis of substrates are expressed in percent hydrolysis of PCG. 